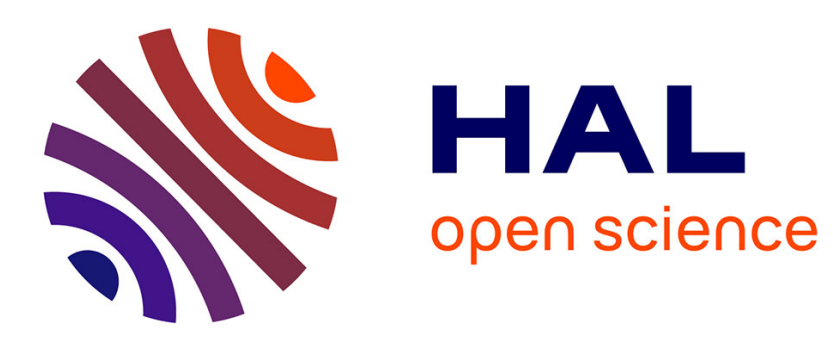

\title{
Velocity-sensorless tracking control and identification of switched-reluctance motors
}

Erik Chumacero, Antonio Loria, G. Espinosa-Pérez

\section{To cite this version:}

Erik Chumacero, Antonio Loria, G. Espinosa-Pérez. Velocity-sensorless tracking control and identification of switched-reluctance motors. Automatica, 2014, 50 (12), pp.3123-3130. hal-00831471

\section{HAL Id: hal-00831471 \\ https://hal.science/hal-00831471}

Submitted on 7 Jun 2013

HAL is a multi-disciplinary open access archive for the deposit and dissemination of scientific research documents, whether they are published or not. The documents may come from teaching and research institutions in France or abroad, or from public or private research centers.
L'archive ouverte pluridisciplinaire $\mathbf{H A L}$, est destinée au dépôt et à la diffusion de documents scientifiques de niveau recherche, publiés ou non, émanant des établissements d'enseignement et de recherche français ou étrangers, des laboratoires publics ou privés. 


\title{
Velocity-sensorless tracking control and identification of switched-reluctance motors
}

\author{
Erik Chumacero $^{a} \quad$ Antonio Loria $^{a b} \quad$ Gerardo Espinosa-Pérez $^{c}$ \\ ${ }^{a}$ LSS-SUPELEC, Univ Paris SUD, ${ }^{b}$ CNRS, 3 Rue Joliot Curie, Gif sur Yvette France. E-mail: chumacero@lss.supelec.fr \\ ${ }^{c}$ DEPFI - UNAM, A.P. 70-256, 04510 México D.F., MEXICO. gerardoe@servidor.unam.mx
}

\begin{abstract}
We present a solution to the speed sensorless control problem for switched-reluctance motors under parametric uncertainty. Our main results guarantee velocity tracking control for velocity references with constant reference acceleration under the assumption that the load torque, the rotor inertia, the resistance and inductances are unknown. Under a persistency of excitation condition on a function which depends only on reference trajectories, we guarantee uniform global asymptotic stability therefore, we establish conditions for the identification of the physical parameters of the system. Our theoretical findings are supported by illustrative simulation results.
\end{abstract}

\section{Introduction}

The fact that switched-reluctance motors are highly reliable and cheap to construct [20] make them very attractive for a number of applications. However, from a modelling viewpoint they are complex machines: e.g., as they are doubly-salient brushless motors [8], it is not possible to apply well-established AC motor rotating field theory; also, the nonuniform nature of electromechanical torque leads to torque ripple. This and their commonly practised operation in magnetic saturation to maximise the torque, make control, modelling and identification of switched-reluctance motors a challenging active research area among various communities.

There exist a large number of efficient heuristically-based and experimentally-validated identification and control approaches for switched-reluctance machines with different control objectives: torque and flux control -[1], velocity control -[5], state estimation -[6], parameters identification -[3], etc. Of particular interest is to reduce the number of mechanical sensors (position and velocity) in the loop as this has a direct impact in the cost of production. For instance, in [5] the authors propose an efficient inductance model-based sensorless control scheme to run the SRM from stand-still to high speed.

Articles on control of switched-reluctance drives that include a rigorous stability analysis, especially in a sensorless context (let alone under parametric uncertainty), are rare. For instance, in [17] there is reported a proportional-derivativebased controller but which relies on the knowledge of the load torque and full-state feedback. The main result in [4] establishes global asymptotic stability for a passivity-based controller in the case of unknown load however, it uses both angular velocity and position measurements. In [13] the authors present an adaptive position-feedback controller which uses an approximate-differentiation filter instead of velocity measurements and it is guaranteed (under a high-gain condition) that the tracking errors converge to zero. However, the model used in [13] comprises only the rotor dynamics and it is assumed that the currents are valid physical control inputs. Although the latter is fully justified, for instance in view of the torque-sharing approach - see [17], the analysis of the stator control loop is not included in the former reference. It is worth remarking that the lack of analysis in a sensorless context is certainly not the case for other electrical machines such as induction motors - see [12, 7], or permanent-magnet synchronous machines -[18, 16]. In [2] a nonlinear controller using a similar scheme is presented however, the authors suppose the mechanical load-toque $\tau_{L}$ known and the model includes viscous friction, which eases considerably the stability analysis.

We present a robust adaptive speed sensorless controller for switched-reluctance motors with uncertainty in all physical parameters. The control scheme is composed of an outer rotor control loop of proportional-integral-derivative type in which the stator currents are considered as virtual control inputs and is robust to the uncertainty in the inertia. Then, an inner adaptive tracking control loop for the stator currents is incorporated. We establish uniform global exponential stability in the case that the stator parameters are known and for the overall adaptively controlled system we establish uniform global asymptotic stability; notably, we establish necessary and sufficient conditions for the convergence of the physical parameter estimates. As far as we know, similar results have not been reported in the literature.

The rest of the paper is organised as follows. In the following section we describe the motor model; in Section 3 we describe the control approach. The formal statements and proofs are provided in Section 4 and some simulations that illustrate our theoretical findings are presented in Section 5, before concluding with some remarks.

\section{The motor model}

Switched-reluctance motors operation is based on the phenomenon that can be observed when two displaced opposite polarised magnets are aligned. The basic structure of a machine with 6 poles in the stator and 4 poles in the rotor

\footnotetext{
1 The work of G. Espinosa is supported by DGAPA-UNAM under grant IN114513. E. Chumacero benefits from a scholarship by CONACyT, Mexico.
} 
is depicted in Figure 1. Each of the symmetrical pole-pairs are simultaneously energised (using a single coil and only positive currents) producing a magnetic field that aligns the winding-free rotor. Mechanical force is produced by exciting, in a suitable way (usually sequentially), the different stator phases.

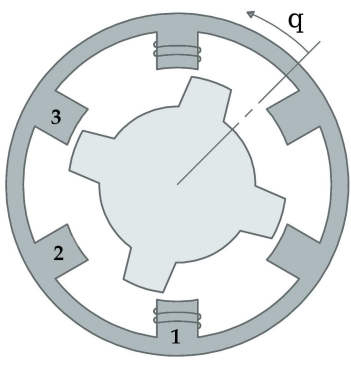

Fig. 1. Diagram of a switched-reluctance motor

After experimental evidence, the three stator phases of a switchedreluctance motor may be assumed to be magnetically decoupled i.e., the mutual inductance among stator phases is negligible. Under such hypothesis an experimentally-validated three-phases dynamic model is proposed in [8]. However, although the model used in the latter reference accounts for magnetic saturation, it defines a nonlinear non-invertible map currents-torque. Therefore, for control and analysis purposes we assume that the flux is linear in the currents that is, the model of the stator dynamics is given, for each phase $j$, by

$$
u_{j}=L_{j}(q) \dot{x}_{j}+K_{j}(q) \omega x_{j}+R x_{j}
$$

where all variables are scalar, $u_{j}$ denotes the voltage applied to the stator terminals, $q$ is the angular rotor position, $\omega$ is the angular velocity, $x_{j}$ denotes the stator current, $R$ denotes the stator winding resistance,

$$
L_{j}(q)=\ell_{0}-\ell_{1} c_{j}, \quad c_{j}:=\cos \left(N_{r} q-(j-1) \frac{2 \pi}{3}\right)
$$

with $\ell_{0}>\ell_{1}>0$, denotes the phase inductance, $N_{r}$ is the number of poles and

$$
K_{j}(q)=\frac{\partial L_{j}}{\partial q}=N_{r} \ell_{1} s_{j}, \quad s_{j}:=\sin \left(N_{r} q-(j-1) \frac{2 \pi}{3}\right),
$$

corresponds to the phase-inductance variation relative to the rotor angular position. Hence, in matrix form, we have

$$
L(q) \dot{x}+K(q) \omega x+R x=u
$$

where $L:=\operatorname{diag}\left\{L_{j}\right\}, K:=\operatorname{diag}\left\{K_{j}\right\}$ and $x=\left[x_{1}, x_{2}, x_{3}\right]^{\top}$. For further development, we remark that there exist constants $\ell_{m}, \ell_{M}$ and $k_{M}$ such that for all ${ }^{2} q \in[-\pi, \pi]$ and $j \in\{1,2,3\}$,

$$
0<\ell_{m} \leq\left\|L_{j}(q)\right\| \leq \ell_{M}, \quad\left\|K_{j}(q)\right\| \leq k_{M}
$$

The rotor dynamics is given by a simple double integrator of the load torque $\tau_{L}$ and the mechanical torque of electrical origin, $\tau_{e}$, which depends on the angular rotor position and the stator currents that is,

$$
\begin{aligned}
J \dot{\omega} & =\tau_{e}(q, x)-\tau_{L} \\
\dot{q} & =\omega
\end{aligned}
$$

where and $J$ is the total rotor inertia. Considering that the behaviour of the stator windings is decoupled, the mechanical torque of electrical origin corresponds to the sum of torques produced by each of the three phases,

$$
\tau_{e}(q, x)=\sum_{j=1}^{3} \frac{1}{2} K_{j}(q) x_{j}^{2} .
$$

\section{Control problem and its solution}

For the system (1), (3) the control problem is to design a dynamic controller whose output $u=\left[\begin{array}{llll}u_{1} & u_{2} & u_{3}\end{array}\right]^{\top}$ depends on the stator currents and rotor angular positions, such that $\omega(t)$ tracks a reference trajectory $\omega^{*}(t)$ under the following standing hypotheses.

\section{Assumption 1}

- The function $\omega^{*}$ is bounded, differentiable and $\dot{\omega}^{*}$ is (piecewise) constant;

- the inertia $J$ is unknown and belongs to an interval of known limits $J_{m}, J_{M}$ i.e., $J \in\left[J_{m}, J_{M}\right]$;

- the load torque $\tau_{L}$ is piece-wise-constant and unknown.

We emphasise that from a control-theory perspective this problem is open.

The control approach consists in applying two control loops: an outer loop to robustly stabilise the rotor dynamics via the virtual control input $\tau_{e}$ and an inner loop to stabilise the stator dynamics using currents measurement to guarantee the tracking control goal $\tau_{e} \rightarrow \tau_{e}^{*}$.

\footnotetext{
${ }^{2}$ Throughout the paper we denote by $\|\cdot\|$, the Euclidean norm of vectors and the induced norm of matrices.
} 


\subsection{Robust control of rotor velocity}

Since the rotor equation (3a) is linear with a (piecewise) constant perturbation $\tau_{L} / J$ and a vanishing disturbance, we chose to use proportional-integral-derivative (PID) control. Since the velocities $\omega$ are not measured we use the $\mathrm{PI}^{2} \mathrm{D}$ controller, introduced in [14] for robot manipulators. Its name comes from the fact that it corresponds to a modified PID controller; it consists in a correction term proportional to the tracking errors $e_{q}$, a 'derivative' term proportional to filtered velocities $\vartheta$ and a double integral action, both on $e_{q}$ and $\vartheta$. Besides its mathematical simplicity, the choice of this controller is motivated by the fact that it conserves the passivity properties of Lagrangian systems and ensures asymptotic stability, provided a property of detectability holds - see [15]. Moreover, since it is a PID controller it is model-free, which is fundamental in the present context in which we assume that the physical parameters are unknown. The $\mathrm{PI}^{2} \mathrm{D}$ tracking controller for the rotor dynamics is defined by

$$
\begin{aligned}
\tau_{d} & =-k_{p} e_{q}-k_{d} \vartheta+\nu+\dot{\omega}^{*} \\
\dot{\nu} & =-k_{i}\left(e_{q}-\vartheta\right) \\
\dot{q}_{c} & =-a\left(q_{c}+b e_{q}\right) \\
\vartheta & =q_{c}+b e_{q}
\end{aligned}
$$

where $k_{p}, k_{i}, k_{d}, a, b$ are positive constants and $e_{q}=q-q^{*}$ with

$$
q^{*}(t)=\int_{0}^{t} q^{*}(s) d s, \quad q^{*}(0)=q_{0}^{*} \in[-\pi, \pi] .
$$

Since the variable to be controlled is $\omega$, the initial value $q^{*}(0)$ is innocuous. Equations (4c) and (4d) correspond to the widely-used "approximate differentiation" filter

$$
\vartheta=\frac{b}{p+a} e_{\omega}
$$

where $p$ is the Laplace variable and $e_{\omega}:=\omega-\omega^{*}$. That is, $\vartheta$ is not an estimate of the velocity $e_{\omega}$ but a filtered version of it; in the limit case, when the pole is at $-\infty, \vartheta=e_{\omega}$ modulo the DC gain $b / a$.

The following is a preliminary result on the stability of the solutions of $(3 \mathrm{a})$ driven by the $\mathrm{PI}^{2} \mathrm{D}$ controller.

Proposition 1 Consider the $P I^{2} D$ controller (4), let $k_{p}^{\prime}:=k_{p}-(k i / \varepsilon)$ where $\varepsilon \in(0,1), k_{i} \leq \varepsilon$ and let

$$
\tau_{e}^{*}=\eta \tau_{d}, \quad \eta>0
$$

Then, the solution

$$
\left\{[q \omega \nu]^{\top}=\left[q^{*} \omega^{*} \nu^{*}\right]\right\}, \quad \nu^{*}:=\frac{\tau_{L}}{\eta}+\left(\frac{J}{\eta}-1\right) \dot{\omega}^{*}
$$

of the system (3a), (3b) with $\tau_{e}=\tau_{e}^{*}$ and under Asssumption 1, is uniformly globally exponentially stable, provided that

$$
A(\eta):=\left[\begin{array}{cccc}
0 & 1 & 0 & 0 \\
-(\eta / J) k_{p}^{\prime} & 0 & -(\eta / J) k_{d} & (\eta / J) \\
0 & b & -a & 0 \\
-k_{i} & -k_{i} / \varepsilon & k_{i} & 0
\end{array}\right]
$$

is Hurwitz. Consequently, if $\tau_{e} \neq \tau_{e}^{*}$ the system is input-to-state stable with input $\tilde{\tau}_{e}:=\tau_{e}-\tau_{e}^{*}$.

Remark 1 The parameter $\eta$ is introduced to underline the robustness of the $\mathrm{PI}^{2} \mathrm{D}$ controller. Indeed, the closed-loop equilibrium for the rotor dynamics depends on the (possibly unknown) parameter $\eta$ but not stability of that equilibrium. Based on [10], standard but somewhat lengthy computations, show that the matrix $A(\eta)$ in $(9)$ is Hurwitz for any $J \in\left[J_{m}, J_{M}\right]$ and $\eta \in\left[\eta_{m}, \eta_{M}\right]$ if

$$
b \geq\left[\frac{\eta_{M}}{J_{m}}+1\right] a+1, \quad k_{p}^{\prime}>k_{d}
$$

Hence, the eigenvalues of $A$ may be made negative by properly choosing the control gains $k_{p}, k_{d}$ and $k_{i}$ without the knowledge of $\eta$ and $J$.

Proof of Proposition 1. In view of (4a) and (5), Equation (3a) is equivalent to

$$
\dot{\omega}=-\frac{\eta}{J}\left[k_{p}^{\prime} e_{q}+\frac{k_{i}}{\varepsilon} e_{q}+k_{d} \vartheta\right]+\frac{\eta}{J}\left(\nu+\dot{\omega}^{*}\right)-\frac{\tau_{L}}{J}+\frac{\tilde{\tau}_{e}}{J} .
$$

Next, let

$$
z:=\nu-\nu^{*}-\frac{k_{i}}{\varepsilon} e_{q}
$$


where $\nu^{*}$ is defined in (6) then,

$$
\dot{e}_{w}=-\frac{\eta}{J}\left[k_{p}^{\prime} e_{q}+k_{d} \vartheta-z\right]+\frac{\tilde{\tau}_{e}}{J}
$$

On the other hand, Equations (4b), (4c) are equivalent to

$$
\dot{\vartheta}=-a \vartheta+b e_{\omega}
$$

so differentiating on both sides of (8), using (4b) and rearranging terms we obtain the rotor closed-loop dynamics ${ }^{3}$

$$
\dot{\xi}=A \xi+B \tilde{\tau}_{e}, \quad \xi:=\left[e_{q} e_{\omega} \vartheta z\right]^{\top}
$$

where $B=\left[\begin{array}{llll}0 & (1 / J) & 0 & 0\end{array}\right]^{\top}$. Now, the Hurwitz property of $A$ is equivalent to the existence of $P=P^{\top}>0$ and $\gamma_{1}>\|P B\|$ such that $-\left(A^{\top} P+P A\right)=Q$ for a given positive definite matrix $Q$, therefore

$$
V_{1}(\xi)=\frac{1}{2} \xi^{\top} P \xi
$$

satisfies

$$
\dot{V}_{1}(\xi) \leq-\frac{1}{2} \xi^{\top} Q \xi+\frac{\gamma_{1}}{J}\|\xi\|\left|\tilde{\tau}_{e}\right| \quad \text { a.e. }
$$

that is, $V_{1}$ is an ISS-Lyapunov function and $\tilde{\tau}_{e}=0$ implies global exponential stability of $\{\xi=0\}$. The result follows observing that $\xi=0$ implies that $[q \omega \nu]^{\top}=\left[q^{*} \omega^{*} \nu^{*}\right]$.

\subsection{Control of the stator dynamics}

Equation (9) is valid if and only if $\tau_{e}^{*}=\eta \tau_{d}$ for a given $\tau_{d}$; this is accomplished by solving $\tau_{e}^{*}\left(q, x^{*}\right)=\eta \tau_{d}$ for $x^{*}$. To that end, we employ the so-called torque sharing technique introduced in [17]. To that end consider the commutation smooth functions ${ }^{4} q \mapsto m_{j}$ such that $\sum_{j=1}^{3} m_{j}(q)=1$ then, in order to satisfy the resulting equation

$$
\frac{1}{2}\left[x_{1}^{* 2} K_{1}(q)+x_{2}^{* 2} K_{2}(q)+x_{3}^{* 2} K_{3}(q)\right]=\eta \tau_{d}\left[m_{1}(q)+m_{2}(q)+m_{3}(q)\right]
$$

we define

$$
x_{j}^{*}=\left[\frac{2 \eta \tau_{d} m_{j}}{N_{r} \ell_{1} s_{j}(q)}\right]^{1 / 2} \quad \forall j \in\{1,2,3\}
$$

where $s_{j}(q)$ may be equal to zero and the quotient above may be negative. For the $x_{j}^{*}$ to be well-posed we exploit the physics of the reluctance machine as in $[8,17]$ and introduce a current-switching policy as follows. Let the sets

$$
\begin{aligned}
& \Theta_{j}^{+}=\left\{q \in[-\pi, \pi]: s_{j}(q) \geq 0\right\} \\
& \Theta_{j}^{-}=\left\{q \in[-\pi, \pi]: s_{j}(q)<0\right\}
\end{aligned}
$$

and let

where

$$
m_{j}(q)=\left\{\begin{array}{lll}
m_{j}^{+}(q) & \text { if } & \tau_{d} \geq 0 \\
m_{j}^{-}(q) & \text { if } & \tau_{d}<0
\end{array}\right.
$$

$$
\begin{aligned}
& m_{j}^{+}(q)>0 \forall q \in \Theta^{+}, m_{j}^{+}(q)=0 \forall q \in \Theta^{-}, \\
& m_{j}^{-}(q)>0 \forall q \in \Theta^{-}, m_{j}^{-}(q)=0 \forall q \in \Theta^{+} .
\end{aligned}
$$

Because the functions $s_{j}$ are sinusoids out of phase by $2 \pi / 3$, for each $q$ and $\tau_{d}$ there always exists (at least) one $j \in\{1,2,3\}$ such that

$$
\frac{\tau_{d} m_{j}(q)}{s_{j}(q)} \in(0, \infty) \text {. }
$$

Furthermore, to make the phase transitions smooth we introduce hysteresis around the switching condition $s_{j}=0$ i.e.,

$$
x_{j}^{*}:= \begin{cases}{\left[\frac{2 \eta}{N_{r} \ell_{1}}\right]^{1 / 2}\left[\frac{\tau_{d} m_{j}(q)}{s_{j}(q)}\right]^{1 / 2}} & \text { if }\left|s_{j}(q)\right|>\delta_{K} \\ 0 & \text { otherwise }\end{cases}
$$

\footnotetext{
${ }^{3}$ Strictly speaking, (9) is defined for almost all $t$, except for the countable number of points where $\ddot{\omega}^{*} \neq 0$ and $\dot{\tau}_{L} \neq 0$ however, the (Carathéodory) solutions are defined for all $t$.

4 Smoothness is assumed for simplicity, piecewise continuity is enough.
} 
where $\delta_{K}$ is the hysteresis design parameter. Under these conditions we have $\tau_{e}^{*}\left(q, x^{*}\right)=\eta \tau_{d}$ so to ensure that $\tau_{e} \rightarrow \eta \tau_{d}$ we must solve the new tracking control problem $x \rightarrow x^{*}$.

The rationale behind the design of the tracking controller for the stator dynamics builds on the observation that under the action of the tracking control law

$$
u=L(q) \dot{x}^{*}+\omega^{*} K(q) x+R x^{*}-k_{p x} e_{x}, \quad e_{x}=x-x^{*},
$$

the origin of the closed-loop equation

$$
L(q) \dot{e}_{x}+\left[R+k_{p x}\right] e_{x}=-K(q) x e_{\omega}
$$

with zero input $\left(e_{\omega} \equiv 0\right)$ is globally exponentially stable, provided that $k_{p x}>0$. This is clear from the fact that (13) is reminiscent of a linear system with stable drift and $L(q)$ is positive definite uniformly in $q$ hence exponential stability of the origin of

is equivalent to that of the origin of

$$
L(q) \dot{e}_{x}=-\left[R+k_{p x}\right] e_{x}
$$

$$
\dot{e}_{x}=-\left[R+k_{p x}\right] e_{x}
$$

Furthermore, the system may be rendered input to state stable from the input $e_{\omega}$ provided that the gain $k_{p x}$ dominates over the input "gain" function $K(q) x$; note that this is feasible since $q$ and $x$ are measured states. Under these conditions one may invoke a small-gain argument to establish global exponential stability of the origin of the closed-loop system (9), (13).

However, the control law (12) is not implementable since $\dot{x}^{*}$ depends on the unmeasured velocity $\omega$ indeed,

$$
\dot{x}_{j}^{*}= \begin{cases}\alpha_{j}\left[\rho_{j}+\delta_{j} e_{\omega}\right] & \text { if }\left|s_{j}(q)\right|>\delta_{K} \\ 0 & \text { otherwise }\end{cases}
$$

where, if $\left|s_{j}(q)\right|>\delta_{K}$,

$$
\begin{aligned}
\alpha_{j} & =\frac{1}{2}\left[\frac{2 \eta}{N_{r} \ell_{1}}\right]^{1 / 2}\left[\frac{m_{j} \tau_{d}}{s_{j}}\right]^{-1 / 2} \\
\rho_{j} & =\frac{m_{j}}{s_{j}}\left[\left(k_{d} a+k_{i}\right) \vartheta-k_{i} e_{q}+\frac{\partial m_{j}}{\partial q} \frac{\tau_{d} \omega^{*}}{m_{j}}\right]-\frac{m_{j} N_{r} c_{j}}{s_{j}^{2}} \tau_{d} \omega^{*} \\
\delta_{j} & =\frac{1}{s_{j}}\left[-m_{j}\left(k_{p}+k_{d} b\right)+\frac{\partial m_{j}}{\partial q} \tau_{d}\right]-\frac{m_{j} N_{r} c_{j}}{s_{j}^{2}} \tau_{d}
\end{aligned}
$$

otherwise, $\alpha_{j}=\rho_{j}=\delta_{j}=0$.

Therefore, we introduce the following control law which is reminiscent of $u$ defined in (12) except that we drop the term $\alpha_{j} \delta_{j} e_{\omega}$ in the definition of $\dot{x}^{*}=\left[\dot{x}_{1}^{*}, \dot{x}_{2}^{*}, \dot{x}_{3}^{*}\right]^{\top}$ that is, let

$$
u=L(q) \alpha \rho+\omega^{*} K(q) x+R x^{*}-k_{p x} e_{x},
$$

where $\alpha=\operatorname{diag}\left\{\alpha_{1}, \alpha_{2}, \alpha_{3}\right\}, \rho=\left[\begin{array}{lll}\rho_{1} & \rho_{2} & \rho_{3}\end{array}\right]^{\top}$ and $\delta=\left[\begin{array}{lll}\delta_{1} & \delta_{2} & \delta_{3}\end{array}\right]^{\top}$. Therefore, (15) is equivalent to

$$
u=L(q) \dot{x}^{*}+\omega^{*} K(q) x+R x^{*}-k_{p x} e_{x}-L(q) \alpha \delta e_{\omega} .
$$

The closed-loop equation (1) with (16) yields

$$
L(q) \dot{e}_{x}=-\left[R+k_{p x}\right] e_{x}-[K(q) x+L(q) \alpha \delta] e_{\omega}
$$

which is also reminiscent of a perturbed linear system with stable drift; in this case the input gain is given by

$$
g(t, x, y):=[K(q(t)) x+L(q(t)) \alpha(t, y) \delta(t, y)]
$$

in which we underline the dependence of $\alpha$ and $\delta$ on $\omega^{*}(t), q(t)$ and the measurable output $y:=\left[e_{q} \vartheta \nu\right]^{\top}$. Moreover, since $L, K, m_{j}, \frac{\partial m_{j}}{\partial q}$ and $\omega^{*}$ are uniformly bounded, there exists a non-decreasing function $\gamma_{2}: \mathbb{R}_{\geq 0} \times \mathbb{R}_{\geq 0} \rightarrow \mathbb{R}_{\geq 0}$ such that

$$
\|g(t, x, y)\| \leq \gamma_{2}(\|y\|,\|x\|) .
$$

Note that $\gamma_{2}$ depends on $\eta_{M} \geq \eta$ but not on the possibly unknown constant $\eta$. Thus, it may be established that (17) is input-to-state stable with respect to the input $e_{\omega}$, for an appropriate choice of the gain $k_{p x}$ depending on $\gamma_{2}$, hence on $\|y\|$ and $\|x\|$. 


\section{Main results}

From the previous developments we see that the closed-loop system (9), (17) consists in the interconnection of two input-to-state stable systems for which the feedback gains may be adjusted to ensure global exponential stability. The proof of this claim constitutes the first of our main contributions.

\subsection{Robust rotor control}

Proposition 2 Consider the system (1), (3) in closed loop with the $P I^{2} D$ controller (4) under the conditions of Proposition 1 and the control law (15) where $x^{*}=\left[\begin{array}{lll}x_{1}^{*} & x_{2}^{*} & x_{3}^{*}\end{array}\right]^{\top}$ is defined via (11) with $\eta:=\hat{J} \in\left[J_{m}, J_{M}\right]$. Let the control gains and $\hat{J}$, which is a constant estimate of $J$, be such that the matrix $A(\hat{J})$ in (7) is Hurwitz for any $J \in\left[J_{m}, J_{M}\right]$ -see Remark 1. Assume that the stator parameters $\ell_{0}, \ell_{1}$ and $R$ are known. Then, there exist a real number $k_{p x}^{\prime}>0$ and a non-decreasing function $k_{p x}^{\prime \prime}: \mathbb{R}_{\geq 0}^{3} \rightarrow \mathbb{R}_{\geq 0}$ such that defining

$$
k_{p x}=k_{p x}^{\prime}+k_{p x}^{\prime \prime}\left(\|y\|,\|x\|,\left\|x^{*}\right\|\right),
$$

with $e_{x}:=x-x^{*}$, the origin of the closed-loop system $\left\{\left[\xi, e_{x}\right]=[0,0]\right\}$ is uniformly globally exponentially stable.

Proof. In view of the definition of $x^{*}$ we have $\tau_{e}^{*}=\hat{J} \tau_{d}$ so the rotor closed-loop dynamics yields (9) with $A=A(\hat{J})$. In view of Proposition 1 the equilibrium defined by (6) is uniformly globally exponentially stable if $\tilde{\tau}_{e}=0$. Let $q_{m}>0$, $\varepsilon \in(0,1)$ and $Q=Q^{\top}>0$ be such that $Q=Q_{1}+Q_{2}$ where $Q_{1}=Q_{1}^{\top}$ is positive definite and $Q_{2}=Q_{2}^{\top}$ is positive semidefinite such that $q_{m}\|\xi\|^{2} \leq \frac{1}{2} \xi^{\top} Q_{1} \xi$ and $\xi^{\top} Q_{2} \xi \geq(J / \eta) \varepsilon(b-1) e_{\omega}^{2}$. Let the Hurwitz assumption generate via [9, Theorem 4.2], a matrix $P$ and positive reals $\gamma_{1}, p_{m}$ and $p_{M}$ such that $Q=-\left(A^{\top} P+P A\right),\|P B\| \leq \gamma_{1}$ and

$$
p_{m}\|\xi\|^{2} \leq V_{1}(\xi) \leq p_{M}\|\xi\|^{2} .
$$

Then, the derivative of $V_{1}$ satisfies (10) with $Q$ as defined above and since $\tau_{e}$ and $\tau_{e}^{*}$ are quadratic functions uniformly bounded in $q, 2\left|\tilde{\tau}_{e}\right| \leq k_{M}\left|e_{x}^{\top}\left(x+x^{*}\right)\right|$ so

$$
\dot{V}_{1}(\xi) \leq-q_{m}\|\xi\|^{2}+\frac{k_{M} \gamma_{1}}{2}\|\xi\|\left|e_{x}^{\top}\left(x+x^{*}\right)\right|-(J / \eta) \varepsilon(b-1) e_{\omega}^{2} \quad \text { a.e. }
$$

On the other hand, substituting $u$ from (15) in (1) we obtain the stator closed-loop equation

$$
L(q) \dot{e}_{x}=-\left[R+k_{p x}\right] e_{x}-g(t, x, y) e_{\omega} .
$$

Consider the function $V_{2}: \mathbb{R}_{\geq 0} \times \mathbb{R}^{3} \rightarrow \mathbb{R}_{\geq 0}$ defined by

$$
V_{2}\left(t, e_{x}\right)=\frac{1}{2} e_{x}^{\top} L(q(t)) e_{x}
$$

which is positive definite and radially unbounded since $\|L(q)\|$ is uniformly bounded and positive definite actually, in view of $(2)$,

$$
\frac{\ell_{m}}{2}\left\|e_{x}\right\|^{2} \leq V_{2}\left(t, e_{x}\right) \leq \ell_{M}\left\|e_{x}\right\|^{2} .
$$

The total derivative of $V_{2}$ along the trajectories of (18) satisfies

$$
\dot{V}_{2}\left(t, e_{x}\right) \leq-e_{x}^{\top}\left[\left(k_{p x}-\gamma_{2}^{2} \lambda_{1}-\frac{k_{M}}{2} \omega^{*}\right) I-\frac{\lambda_{3}}{2} e_{x}^{\top} K(q(t)) e_{x} K(q(t))\right] e_{x}+\left[\frac{1}{\lambda_{1}}+\frac{1}{2 \lambda_{3}}\right] e_{\omega}^{2}
$$

for which we used $\frac{d}{d t} L(q(t))=K(q(t))\left[e_{\omega}+\omega^{*}\right], e_{x}^{\top} K(q) e_{x} e_{\omega} \leq\left(1 / \lambda_{3}\right) e_{\omega}^{2}+\lambda_{3}\left[e_{x}^{\top} K(q) e_{x}\right]^{2}$ and $e_{x}^{\top} g e_{\omega} \leq \lambda_{1} \gamma_{2}^{2}\left\|e_{x}\right\|^{2}+$ $\left(1 / \lambda_{1}\right) e_{\omega}^{2}$. Thus, the total derivative of the Lyapunov function $V_{3}:=V_{1}+V_{2}$ along the closed-loop trajectories satisfies

$$
\begin{aligned}
\dot{V}_{3} \leq- & {\left[\left(J_{m} / \eta_{M}\right) \varepsilon(b-1)-\frac{1}{\lambda_{1}}-\frac{1}{2 \lambda_{3}}\right] e_{\omega}^{2}-\left[q_{m}-\frac{\lambda_{2} k_{M} \gamma_{1}}{2}\right]\|\xi\|^{2} } \\
& -\left[k_{p x}-\lambda_{1} \gamma_{2}^{2}-\frac{k_{M}}{2}\left(\omega^{*}+\lambda_{3} k_{M}\left\|e_{x}\right\|^{2}+\frac{\gamma_{1}}{\lambda_{2}}\left\|x+x^{*}\right\|^{2}\right)\right]\left\|e_{x}\right\|^{2} \quad \text { a.e. }
\end{aligned}
$$

where we used $\left|e_{x}^{\top}\left(x+x^{*}\right)\right|\|\xi\| \leq\left(1 / \lambda_{2}\right)\left\|x+x^{*}\right\|^{2}+\lambda_{2}\|\xi\|^{2}$. Therefore, given $q_{m}$ and $b$, for any $J, \eta$ and $\varepsilon$, there exist positive numbers $\lambda_{1}, \lambda_{2}, \lambda_{3}$ and $c$ such that, defining $k_{p x}^{\prime}>0$ and

$$
k_{p x}^{\prime \prime}:=\frac{k_{M}}{2}\left(\omega^{*}+\lambda_{3} k_{M}\left\|e_{x}\right\|^{2}+\frac{\gamma_{1}}{\lambda_{2}}\left\|x+x^{*}\right\|^{2}\right)+\lambda_{1} \gamma_{2}(\|y\|,\|x\|)^{2},
$$


$V_{3}$ satisfies

$$
\dot{V}_{3}\left(t, \xi, e_{x}\right) \leq-c\left[\|\xi\|^{2}+\left\|e_{x}\right\|^{2}\right] \quad \text { a.e. }
$$

Note that $k_{p x}^{\prime \prime}$ is a non-decreasing function of $\|y\|,\|x\|$ and $\left\|x^{*}\right\|$ since $\left\|x^{*}\right\|^{2}=\mathcal{O}\left(\tau_{d}\right)$ where $\tau_{d}$ is linear in $y$. The result follows.

\subsection{Control under full parametric uncertainty}

Now we assume that in addition to $J$ and $\tau_{L}$, the physical parameters $\ell_{0}, \ell_{1}$ and $R$ are also unknown. Let $C(q):=$ $\operatorname{diag}\left\{c_{j}(q)\right\}, S(q):=\operatorname{diag}\left\{s_{j}(q)\right\}$ then, $L(q)=\ell_{0} I-\ell_{1} C(q)$ and $K(q)=\ell_{1} N_{r} S(q)$. We introduce the constant estimate of $\ell_{1}, \hat{\ell}_{1 \circ} \in\left[\ell_{m}, \ell_{M}\right]$ to redefine $\eta$ in $(11)$ as

$$
\eta:=\frac{\hat{J} \ell_{1}}{\hat{\ell}_{10}}
$$

so $\alpha$ in (14) and $x^{*}$ in (11) depend only on known quantities. With this notation, the control law (15) may be written as

$$
u=\ell_{0} \alpha \rho-\ell_{1} C(q) \alpha \rho+\omega^{*} \ell_{1} N_{r} S(q) x+R x^{*}-k_{p x} e_{x}
$$

which is linear in the physical parameters $\ell_{0}, \ell_{1}$ and $R$ therefore,

$$
\begin{aligned}
& u=\Psi\left(t, y, e_{x}\right)^{\top} \Theta-k_{p x} e_{x}, \\
& \Psi\left(t, y, e_{x}\right)^{\top}:=\left[\begin{array}{lll}
\alpha \rho & \omega^{*} N_{r} S(q) x-C(q) \alpha \rho & x^{*}
\end{array}\right], \\
& \Theta:=\left[\begin{array}{lll}
\ell_{0} \ell_{1} R & ]^{\top} .
\end{array}\right.
\end{aligned}
$$

We stress that $\Psi$ is a function of $t$, the closed-loop states $e_{x}$ and the measured outputs $y=\left[e_{q} \vartheta \nu\right]^{\top}$; indeed, one should read $q(t)$ and $x=e_{x}+x^{*}$ in place of $q$ and $x$ while $\alpha, \rho$ and $x^{*}$ are functions of $t, y$ and known constants.

Proposition 3 Consider the system (1), (3) in closed loop with the PI D controller (4) under the conditions of Proposition 1 and

$$
\begin{aligned}
& u=\Psi\left(t, y, e_{x}\right)^{\top} \hat{\Theta}-k_{p x} e_{x}, \\
& \dot{\hat{\Theta}}=-k_{\theta} \Psi\left(t, y, e_{x}\right) e_{x}, \quad k_{\theta}>0
\end{aligned}
$$

Let the control gains and $\hat{J} \in\left[J_{m}, J_{M}\right]$ be such that the matrix $A(\eta)$, with $\eta$ as in (19), is Hurwitz for any $J \in\left[J_{m}\right.$, $\left.J_{M}\right]$. Define $\tilde{\Theta}:=\hat{\Theta}-\Theta$. Then, the origin of the closed-loop system, $\left\{\left[\xi, e_{x}, \tilde{\Theta}\right]=[0,0,0]\right\}$ is uniformly globally stable (i.e., the origin is uniformly stable and the solutions are uniformly globally bounded) and the tracking errors $\xi$ and $e_{x}$ satisfy

$$
\lim _{t \rightarrow \infty}\|\xi(t)\|=0 \quad \lim _{t \rightarrow \infty}\left\|e_{x}(t)\right\|=0
$$

Proof. A direct computation using

$$
u=\Psi\left(t, y, e_{x}\right)^{\top} \Theta-k_{p x} e_{x}+\Psi\left(t, y, e_{x}\right)^{\top} \tilde{\Theta}
$$

leads to the closed-loop equations

$$
\begin{aligned}
L(q) \dot{e}_{x} & =\left[R+k_{p x}\right] e_{x}-g(t, x, y) e_{\omega}+\Psi\left(t, y, e_{x}\right)^{\top} \tilde{\Theta} \\
\dot{\tilde{\Theta}} & =-k_{\theta} \Psi\left(t, y, e_{x}\right) e_{x} .
\end{aligned}
$$

Next, consider the Lyapunov function $V: \mathbb{R}_{\geq 0} \times \mathbb{R}^{4} \times \mathbb{R}^{3} \times \mathbb{R}^{3} \rightarrow \mathbb{R}_{\geq 0}$ defined by

$$
V\left(t, \xi, e_{x}, \tilde{\Theta}\right):=V_{3}\left(t, \xi, e_{x}\right)+\frac{1}{2 k_{\theta}}\|\tilde{\Theta}\|^{2}
$$

which is positive definite and radially unbounded. The Hurwitz property of $A$ implies by Proposition 2 that the total derivative of $V$ along the closed-loop trajectories generated by (22) satisfies

$$
\dot{V}\left(t, \xi, e_{x}, \tilde{\Theta}\right) \leq-c\left[\|\xi\|^{2}+\left\|e_{x}\right\|^{2}\right] \leq 0 \quad \text { a.e. }
$$

Uniform global stability of the origin follows integrating $\dot{V} \leq 0$ along the closed-loop trajectories hence $\xi \in \mathcal{L}_{\infty}, e_{x} \in \mathcal{L}_{\infty}$ and, in view of (2), a simple inspection at the closed-loop equations show that $\dot{\xi} \in \mathcal{L}_{\infty}$ and $\dot{e}_{x} \in \mathcal{L}_{\infty}$. Furthermore, the first inequality in (23) implies that $\xi \in \mathcal{L}_{2}, e_{x} \in \mathcal{L}_{2}$. The result follows from Barbalăt's lemma.

Under an additional condition of persistency of excitation, one may establish the uniform convergence of the parameter estimation errors. 
Proposition 4 Consider the system (1), (3) in closed loop with the PI D controller (4) under the conditions of Proposition 1 and (21) under the conditions of Proposition 3. Then, the origin of the closed loop system is uniformly globally asymptotically stable if and only if $\Psi_{0}(t):=\Psi(t, 0,0)$ is persistently exciting that is if there exist $\mu>0$ and $T>0$ such that

$$
M(t):=\int_{t}^{t+T} \Psi_{0}(s) \Psi_{0}(s)^{\top} d s \geq \mu I \quad \forall t \geq 0 .
$$

Proof. The proof of Proposition 4 follows by applying [11, Theorem 1]. The first condition of the latter is that the origin is uniformly globally stable, this is established in Proposition 3 . From the proof of the latter, we have $\dot{V} \leq Y_{0}\left(\xi, e_{x}\right)$ a.e. with $Y_{0}\left(\xi, e_{x}\right)=-c\left[\|\xi\|^{2}+\left\|e_{x}\right\|^{2}\right] \leq 0$. It is left to find differentiable locally bounded auxiliary functions with the property that their derivatives are negative semi-definite on certain sets. The first auxiliary function must be negative semi-definite on $\left\{Y_{0}=0\right\}$ hence, we look for a function $W_{1}: \mathbb{R}_{\geq 0} \times \mathbb{R}^{4} \times \mathbb{R}^{3} \times \mathbb{R}^{3} \rightarrow \mathbb{R}$ such that

$$
\left[\xi, e_{x}\right]=[0,0] \Longrightarrow \dot{W}_{1} \leq 0
$$

We propose

$$
W_{1}\left(t, \xi, e_{x}, \tilde{\Theta}\right)=-e_{x}^{\top} L(q(t))^{\top} \Psi_{0}(t)^{\top} \tilde{\Theta}
$$

whose total derivative along the closed-loop trajectories generated by (22) yields

$$
\begin{aligned}
\dot{W}_{1}\left(t, \xi, e_{x} \tilde{\Theta}\right)=- & e_{x}^{\top}\left(k_{p x}+R\right) \Psi_{0}(t)^{\top} \widetilde{\Theta}-g(t, x, \xi)^{\top} \Psi_{0}(t)^{\top} \tilde{\Theta} e_{\omega}-\widetilde{\Theta}^{\top} \Psi\left(t, y, e_{x}\right) \Psi_{0}(t)^{\top} \widetilde{\Theta} \\
& -e_{x}^{\top} \dot{L}(q(t))^{\top} \Psi_{0}(t)^{\top} \widetilde{\Theta}-e_{x}^{\top} L(q(t))^{\top} \dot{\Psi}_{0}(t) \widetilde{\Theta}+k_{\theta} e_{x}^{\top} L(q(t))^{\top} \Psi_{0}(t)^{\top} \Psi\left(t, y, e_{x}\right) e_{x} .
\end{aligned}
$$

Let $\Omega \subset \mathbb{R}^{10}$ be a compact set; we see that for all $\left(t,\left[\xi, e_{x}, \tilde{\Theta}\right]\right) \in \mathbb{R}_{\geq 0} \times \Omega \cap\left\{Y_{0}=0\right\}$ we have

$$
\begin{aligned}
\dot{W}_{1}\left(t, \xi, e_{x} \tilde{\Theta}\right) & =Y_{1}(t, \tilde{\Theta}) \leq 0 \\
Y_{1}(t, \tilde{\Theta}) & =-\tilde{\Theta}^{\top} \Psi_{0}(t) \Psi_{0}(t)^{\top} \tilde{\Theta}
\end{aligned}
$$

Next, we look for a second auxiliary function $W_{2}$ such that its derivative is negative semi-definite on $\mathbb{R}_{\geq 0} \times \Omega \cap\left\{Y_{0}=\right.$ $0\} \cap\left\{Y_{1}=0\right\}$. Let $W_{2}: \mathbb{R}_{\geq 0} \times \mathbb{R}^{3} \rightarrow \mathbb{R}_{\geq 0}$ be defined by

$$
W_{2}(t, \tilde{\Theta})=-\int_{t}^{\infty} e^{t-\tau}\|\Phi(\tau, \tilde{\Theta})\|^{2} d \tau
$$

where $\Phi(t, \tilde{\Theta})=\Psi_{0}(t)^{\top} \tilde{\Theta}$. Note that

and considering (24),

$$
\int_{t}^{\infty} e^{t-\tau}\|\Phi(\tau, \tilde{\Theta})\|^{2} d \tau \geq \int_{t}^{t+T} e^{t-\tau}\|\Phi(\tau, \tilde{\Theta})\|^{2} d \tau
$$

so we obtain

$$
\int_{t}^{t+T} e^{t-(t+T)}\|\Phi(\tau, \tilde{\Theta})\|^{2} d \tau \geq \mu e^{-T}\|\tilde{\Theta}\|^{2}
$$

$$
W_{2}(t, \tilde{\Theta}) \leq-\mu e^{-T}\|\tilde{\Theta}\|^{2} .
$$

On the other hand, the total time derivative of $W_{2}$ along the closed-loop trajectories satisfies, after (22b) and (25),

$$
\dot{W}_{2}(t, \tilde{\Theta}) \leq 2 k_{\theta} \int_{t}^{\infty} e^{t-\tau} \tilde{\Theta}^{\top} \Psi_{0}(\tau) \Psi_{0}(\tau)^{\top} d \tau \Psi\left(t, y, e_{x}\right) e_{x}+\|\Phi(t, \tilde{\Theta})\|^{2}-\mu e^{-T}\|\widetilde{\Theta}\|^{2} .
$$

Now, we have $\left\{Y_{0}=0\right\} \cap\left\{Y_{1}=0\right\}=\left\{\left[\xi, e_{x}, \Phi\right]=[0,0,0]\right\}$ hence, on this set and for all $t \in \mathbb{R}_{\geq 0}$,

$$
\dot{W}_{2}(t, \tilde{\Theta}) \leq-\mu e^{-T}\|\widetilde{\Theta}\|^{2}
$$

The functions $\dot{V}, \dot{W}_{1}$ and $\dot{W}_{2}$ are bounded from above by continuous functions which vanish simultaneously only at $\left[\xi, e_{x}, \Phi, \tilde{\Theta}\right]=[0,0,0,0]$. The result follows invoking Theorem 1 of $[11]$

\section{Simulation Results}

With aim at evaluating the controller of Proposition 4 we performed some numerical simulations in SIMULINK ${ }^{\mathrm{TM}}$ of MATLAB $^{\mathrm{TM}}$, using the following parameters, taken from [8], $R=0.3[\Omega], \ell_{0}=24[\mathrm{mH}], \ell_{1}=19[\mathrm{mH}]$ and $N_{r}=25$, while the control gains are set to $a=1500, b=3200, k_{p}=1050, k_{i}=5 \mathrm{e}^{-4}, k_{d}=1000, k_{p x}^{\prime}=250, k_{p x}^{\prime \prime}=0, \eta=13 \mathrm{e}^{-4}$ and the adaptive law gain to $k_{\theta}=\operatorname{diag}\left[1 \mathrm{e}^{-4}, 1 \mathrm{e}^{-6}, 2.5 \mathrm{e}^{-5}\right]$. We stress that for implementation purpose, we use a constant value of $k_{p x}$ even though the sufficient condition previously established for global stability is that this gain depends on the measured states. The reason to fix $k_{p x}^{\prime \prime}=0$ is to avoid high values in the input voltages which drive the converters into saturation. 
The simulation consists in imposing a realistic speed reference namely, the desired motor speed starts off from zero, accelerating at $36\left[\mathrm{rad} / \mathrm{s}^{2}\right]$ up to $90[\mathrm{rad} / \mathrm{s}]$. This value is kept constant for other $10 \mathrm{~s}$ until $t=12.5 \mathrm{~s}$ when a new constant reference velocity, of $180[\mathrm{rad} / \mathrm{s}]$, is given to be reached in $0.5 \mathrm{~s}$. The new velocity reference is kept up to $\mathrm{t}=17.5 \mathrm{~s}$ when the motor is rapidly brought to a regime of inverse rotation at $90[\mathrm{rad} / \mathrm{s}]$. During the first $5 \mathrm{~s}$, the load torque equals to $1[\mathrm{Nm}]$ and it is abruptly increased by $50 \%$ at $t=55 \mathrm{~s}$

The commutation functions $m_{j}:[0,2 \pi) \rightarrow \mathbb{R}_{\geq 0}$ which are illustrated with the currents in Figure 3 , are defined as follows. Let

$$
f(x)=\frac{10 x^{3}}{\left(\pi / N_{r}\right)^{3}}-\frac{15 x^{4}}{\left(\pi / N_{r}\right)^{4}}+\frac{6 x^{5}}{\left(\pi / N_{r}\right)^{5}}
$$

and $q_{1}:=\bmod \left(q, 2 \pi / N_{r}\right), q_{2}:=\bmod \left(q-2 \pi / 3 N_{r}, 2 \pi / N_{r}\right), q_{3}:=\bmod \left(q+2 \pi / 3 N_{r}, 2 \pi / N_{r}\right)$ where the operator mod resets $q$ that is, $q_{j}=\bmod \left(\beta_{1}, \beta_{2}\right)$ takes the initial value $q_{j}(0)=\beta_{1}$ and is reset to the latter when $q_{j}(t)=\beta_{2}$. Then,

$$
m_{j}^{+}(q)=\left\{\begin{array}{ll}
f_{j}\left(q_{j}\right) & 0<q_{j} \leq \frac{\pi}{3 N_{r}} \\
1 & \frac{\pi}{3 N_{r}}<q_{j} \leq \frac{2 \pi}{3 N_{r}} \\
1-f_{j}\left(q_{j}\right) \frac{2 \pi}{3 N_{r}}<q_{j} \leq \frac{\pi}{N_{r}} \\
0
\end{array} \quad m_{j}^{-}(q)= \begin{cases}f_{j}\left(q_{j}\right) & \frac{\pi}{N_{r}}<q_{j} \leq \frac{4 \pi}{3 N_{r}} \\
1 & \frac{4 \pi}{3 N_{r}}<q_{j} \leq \frac{5 \pi}{3 N_{r}} \\
1-f_{j}\left(q_{j}\right) & \frac{5 \pi}{3 N_{r}}<q_{j} \leq \frac{2 \pi}{N_{r}} \\
0 & \text { otherwise }\end{cases}\right.
$$

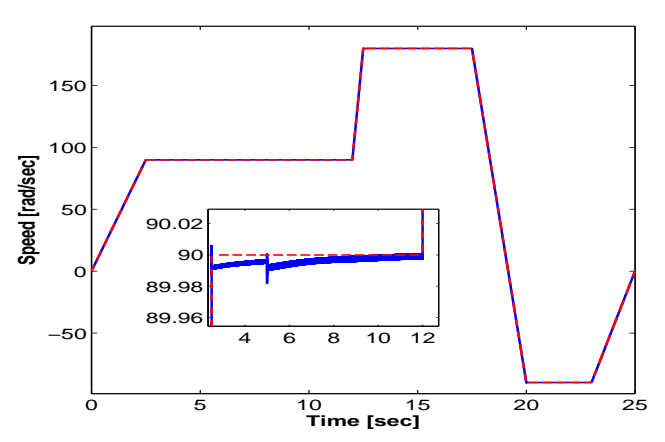

Fig. 2. Velocity Tracking

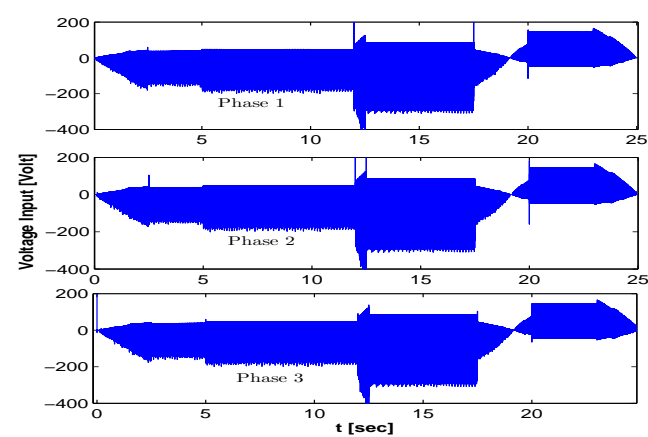

Fig. 4. Voltage control inputs $u_{j}$

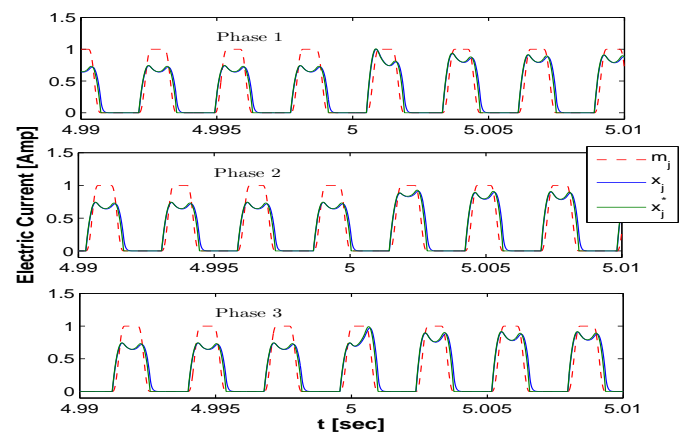

Fig. 3. Electric current around $t=5 \mathrm{~s}$ when the load torque changes. The dashed lines correspond to the commutaion signals $m_{j}$.

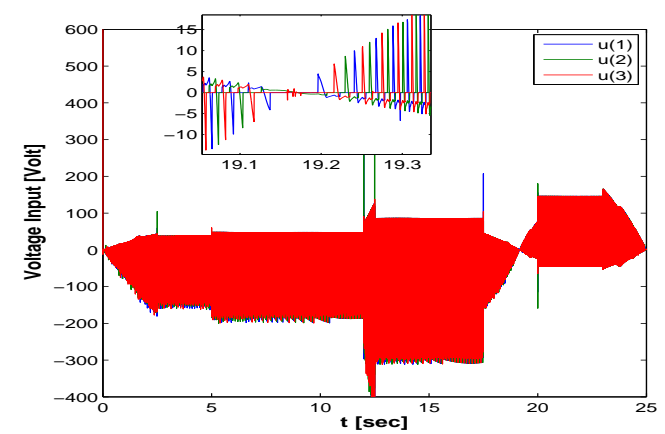

Fig. 5. Voltage control inputs $u_{j}$

The velocity response, which is the variable of interest, is depicted in Figure 2; one may appreciate a perfect tracking of the angular velocity and good performance. The tracking performance for the three currents is illustrated in Figure 3. The latter depicts, on a zoomed window, the three stator currents and their references, driven by the commutation signals $m_{j}(q(t))$; one may appreciate the phase difference of the commutation signals which alternately activate each phase current to inject the virtual control $\tau_{d}$ into the mechanical subsystem. Notice also the effect of the sudden loadtorque increase at $t=5 \mathrm{~s}$ which results in a change of current reference i.e., the motor requires more electrical current in order to remain at the required velocity set-point $-c f$. zoomed window in Figure 2. Back to Figure 3 one may appreciate that the reference current is tracked by the actual currents without delay, in spite of the step. The input voltages (physical control inputs) are depicted in Figures 4 and 5.

The last two figures illustrate the performance of the adaptation law. In Figure 6 are illustrated the responses of the estimated inductance parameters $\ell_{0}, \ell_{1}$ and the estimated resistance $R$, which converge to their true values. Finally, Figure 7 depicts the eigenvalues of $M(t)$ in $(24)$ which are clearly positive on the simulation window, this illustrates that the condition of persistency of excitation is satisfied. 

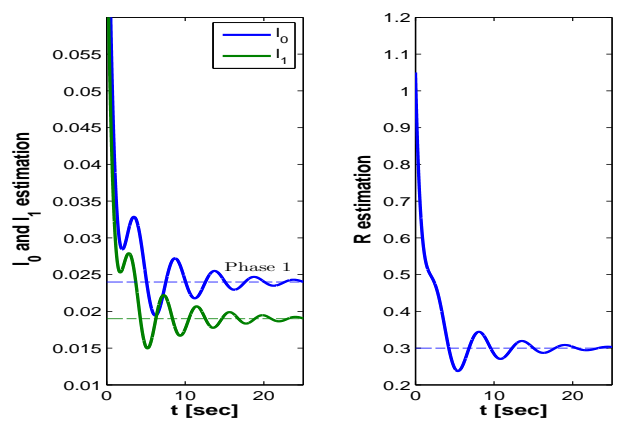

Fig. 6. Estimated parameters converging to the true values

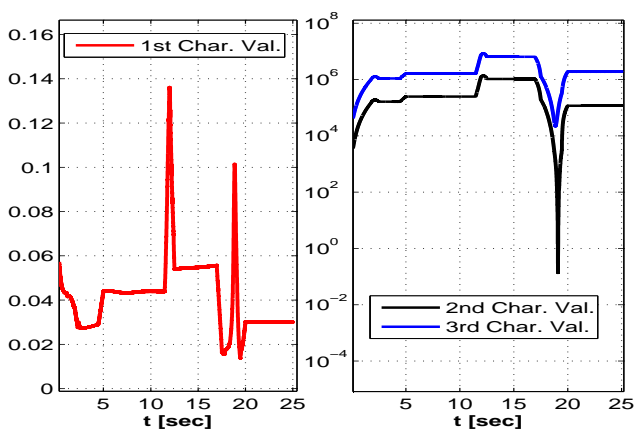

Fig. 7. Verification of the condition of persistency of excitation: eigenvalues of $M(t)$ in (24) on the window of simulation.

\section{Concluding Remarks}

We presented a robust adaptive controller for the switched-reluctance motor, considering both the stator and rotor dynamics. We employ fairly simple control laws (PID, approximate differentiation), widely used in control practise. Although our results guarantee robustness with respect to bounded disturbances (possibly unmodelled dynamics) it is of utmost importance in motor control, to design controllers with guaranteed performance. In that regard, an interesting avenue of research is to exploit more modern adaptation techniques which palliate side-effects such as sensitivity to noise by introducing the so-called $\sigma$-modification. The recent paper [19] presents promising results for model-referenceadaptive control whose applicability (modulo necessary modifications) to motor control, is interesting to analyse. Further ongoing research focuses on the sensorless control problem that is, avoiding the use of position measurements.

\section{References}

[1] A.D. Cheok and Y. Fukuda. A new torque and flux control method for switched reluctance motor drives. Power Electronics, IEEE Transactions on, 17(4):543-557, jul 2002.

[2] Jun Hu D. Dawson and T. Burg. Nonlinear control of electric machinery. Marcel Dekker Inc, 1998.

[3] L.L.N. dos Reis, F. Sobreira, A.R.R. Coelho, O.M. Almeida, J.C.T. Campos, and S. Daher. Identification and adaptive speed control for switched reluctance motor using DSP. In Power Electronics Conference, 2009. COBEP '09. Brazilian, pages 836 -841, 27 2009-oct. 12009.

[4] G. Espinosa-Perez, P. Maya-Ortiz, M. Velasco-Villa, and H. Sira-Ramirez. Passivity-based control of switched reluctance motors with nonlinear magnetic circuits. Control Systems Technology, IEEE Transactions on, 12(3):439 - 448, may 2004.

[5] Hongwei Gao, F.R. Salmasi, and M. Ehsani. Inductance model-based sensorless control of the switched reluctance motor drive at low speed. Power Electronics, IEEE Transactions on, 19(6):1568 - 1573, nov. 2004.

[6] I. Husain and M.S. Islam. Observers for position and speed estimations in switched reluctance motors. In Decision and Control, 2001. Proceedings of the 40th IEEE Conference on, volume 3, pages $2217-2222$ vol.3, 2001.

[7] S. Ibarra-Rojas, J. Moreno, and G. Espinosa-Pérez. Global observability analysis of sensorless induction motors. Automatica, 40(6):1079 - 1085, 2004.

[8] M. Ilic-Spong, R. Marino, S. Peresada, and D. Taylor. Feedback linearizing control of switched reluctance motors. IEEE Trans. Automat. Contr., AC-32:371-379, 1987.

[9] H. Khalil. Nonlinear systems. Prentice Hall, 3rd ed., New York, 2002.

[10] A. Loria, G. Espinosa, and E. Chumacero. Speed-sensorless control of switched-reluctance motors with uncertain payload. In Proc. IEEE American Control Conference, Washington, D.C., 2013. To appear.

[11] A. Loría E. Panteley, D. Popovic, and A. Teel. A nested Matrosov theorem and persistency of excitation for uniform convergence in stable non-autonomous systems. IEEE Trans. on Automat. Contr., 50(2):183-198, 2005.

[12] R. Marino, P. Tomei, and C.M. Verrelli. Nonlinear tracking control for sensorless induction motors. In Decision and Control, 2004. CDC. 43rd IEEE Conference on, volume 4, pages $4423-4428$ Vol.4, dec. 2004.

[13] R. Milman and S.A. Bortoff. Observer-based adaptive control of a variable reluctance motor: Experimental results. Control Systems Technology, IEEE Transactions on, 7(5):613 -621, sep 1999.

[14] R. Ortega, A. Loría and R. Kelly. A semiglobally stable output feedback PI2D regulator for robot manipulators. IEEE Trans. on Automat. Contr., 40(8):1432-1436, 1995.

[15] R. Ortega, A. Loría P. J. Nicklasson, and H. Sira-Ramírez. Passivity-based Control of Euler-Lagrange Systems: Mechanical, Electrical and Electromechanical Applications. Series Comunications and Control Engineering. Springer Verlag, London, 1998. ISBN 1-85233-016-3.

[16] D. Shah, G. Espinosa-Pérez, R. Ortega, and M. Hilairet. An asymptotically stable sensorless speed controller for non-salient permanent magnet synchronous motors. Int. J. Robust Nonlinear Control, 2012. DOI: 10.1002/rnc.2910.

[17] D. Taylor. Pulse-width modulated control of electromechanical systems. IEEE Trans. Automat. Contr., AC-37:524-528, 1992.

[18] P. Tomei and C.M. Verrelli. Observer-based speed tracking control for sensorless permanent magnet synchronous motors with unknown load torque. Automatic Control, IEEE Transactions on, 56(6):1484 -1488, june 2011.

[19] T. Yucelen and W.M. Haddad. Low-frequency learning and fast adaptation in model reference adaptive control. Automatic Control, IEEE Transactions on, 58(4):1080-1085, 2013.

[20] S.W. Zhao, N.C. Cheung, W.C. Gan, J.M. Yang, and Q. Zhong. Passivity-based control of linear switched reluctance motors with robustness consideration. Electric Power Applications, IET, 2(3):164-171, 2008. 